Georgia State University

ScholarWorks @ Georgia State University

1997

\title{
Intrathecal Urokinase as a treatment for intraventricular hemorrhage in the preterm infant
}

\author{
Roger J. Hudgins \\ William R. Boydston \\ Emory University, william.r.boydston@emory.edu \\ Patricia A. Hudgins \\ Emory University, phudgin@emory.edu \\ Robin Morris \\ Georgia State University, robinmorris@gsu.edu \\ Saul M. Adler
}

See next page for additional authors

Follow this and additional works at: https://scholarworks.gsu.edu/psych_facpub

Part of the Psychology Commons

\section{Recommended Citation}

Hudgins, R. J., Boydston, W.R., Hudgins, P.A., Morris, R., Adler, S.M. \& Gilreath, C.L. (1997). Intrathecal Urokinase as a treatment for intraventricular hemorrhage in the preterm infant. Pediatric Neurosurgery, 26; 281-287.

This Article is brought to you for free and open access by the Department of Psychology at ScholarWorks @ Georgia State University. It has been accepted for inclusion in Psychology Faculty Publications by an authorized administrator of ScholarWorks @ Georgia State University. For more information, please contact scholarworks@gsu.edu. 


\section{Authors}

Roger J. Hudgins, William R. Boydston, Patricia A. Hudgins, Robin Morris, Saul M. Adler, and Carita Lynn Gilreath 
Pediatric
Neurosurgery

\section{Roger J. Hudgins a}

William R. Boydston a

Patricia A. Hudgins ${ }^{\mathrm{b}}$

Robin Morris ${ }^{\mathrm{c}}$

Saul M. Adler ${ }^{\mathrm{d}}$

Carita Lynn Gilreath $^{\mathrm{a}}$

a Department of Pediatric Neurosurgery, Scottish Rite Children's Medical Center,

b Department of Radiology,

Emory University School of Medicine,

c Department of Psychology,

Georgia State University, and

d Department of Neonatology,

Northside Hospital, Atlanta, Ga., USA

\section{Intrathecal Urokinase as a Treatment for Intraventricular Hemorrhage in the Preterm Infant}

\section{Key Words}

Intraventricular hemorrhage

Urokinase

Posthemorrhagic hydrocephalus

Prematurity

\section{Abstract}

Despite improvements in the care of preterm infants, intraventricular hemorrhage (IVH) and posthemorrhagic hydrocephalus ( $\mathrm{PHH})$ continue to be frequent occurrences in this patient population. Shunt procedures in these children are frequently complicated by obstruction and/or infection. As the hydrocephalus is usually caused by an obliterative arachnoiditis due to contact of the blood with the basilar meninges, it was postulated that infusion of urokinase into the ventricles of infants who have sustained an IVH would clear the blood, mitigate the arachnoiditis, and prevent the progression of PHH.

Accordingly, 18 preterm infants who had sustained IVH and subsequently developed $\mathrm{PHH}$ were treated with intraventricular urokinase instilled via a surgically implanted subcutaneous reservoir. There were no complications associated with the urokinase. Infants were divided into two dosage groups: low dose (110,000-140,000 IU total) and high dose (280,000 IU total). One infant in the low-dose group died at 1 month of life of respiratory complications. In the low-dose group, 3 of 8 (37\%) infants required shunt placement; in the high-dose group, all 9 required shunt placement. For the total group, the shunt rate was $71 \%$. This compares to a historical control group shunt rate of $92 \%$. While the difference between the treatment group as a whole and control group approaches, but does not reach, statistical significance $(p=0.068)$, there was a significant reduction in the shunt rate when the low-dose group was considered separately $(\mathrm{p}<0.002)$. For those infants that required shunt placement, there were fewer shunt revisions performed in the treatment group than in the control group during the first 24 months following shunt placement: 0.67 versus 1.5 shunt revisions/shunted child.

Initial experience with intraventricular urokinase following IVH and $\mathrm{PHH}$ in preterm infants suggests a beneficial effect in reducing the shunt revision rate in both high- and low-dose groups. Reduction in shunt placement rate is seen only in the low-dose group.

\section{KARGER}

$\mathrm{Fax}+41613061234$ E-Mail karger@karger.ch www. karger.com
Roger J. Hudgins, MD

5455 Meridian Mark Rd, Suite 540

Atlanta, GA 30342 (USA)

Tel +14042556509 , Fax +14042551686 


\section{Introduction}

Intraventricular hemorrhage (IVH) is a common complication of prematurity, occurring in $40-45 \%$ of premature infants weighing $<1,500 \mathrm{~g}$ [1]. Posthemorrhagic hydrocephalus (PHH) develops in $65-100 \%$ of infants with severe IVH, the incidence correlating directly with the severity of the hemorrhage. Many infants who develop $\mathrm{PHH}$ will require placement of a ventricular shunt. Shunt placement, while a very common procedure, is not without risks including cerebral injury, intracranial hemorrhage, and the risks of anesthesia. Shunt infection is a dreaded complication that occurs in approximately $5 \%$ of cases [2] and may have a deleterious effect on the child's intellectual outcome [3]. Shunt obstruction is an ongoing problem and may precipitate several additional surgeries during the life of the child.

The hydrocephalus that follows IVH is caused by an obliterative arachnoiditis in the posterior fossa where the blood tends to pool [4]. The arachnoiditis develops over a period of several days to weeks after the hemorrhage, thus offering a window of opportunity for treatment. Urokinase is a thrombolytic agent that, when placed in the ventricle of the premature infant following IVH, may clear the blood, mitigate the development of arachnoiditis, and prevent the progression of $\mathrm{PHH}$. In addition, as fibrin debris is usually the obstructing agent following shunt placement for $\mathrm{PHH}$, urokinase may facilitate the clearance of the fibrin and decrease the incidence of shunt revision in those infants who do require a shunt following urokinase treatment.

To evaluate safety and efficacy, we have treated 18 premature infants with intraventricular urokinase following onset of $\mathrm{PHH}$.

\section{Materials and Methods}

The study was approved by the Investigational Review Boards of both Scottish Rite Children's Medical Center (SRCMC) and Northside Hospital (NSH).

\section{Patient Population}

From October 1992 to November 1994, 20 infants with IVH and progressive $\mathrm{PHH}$ met the criteria for entry into this study. Two families declined treatment with urokinase leaving a study group of 18 infants. Informed, written consent was obtained from the parents in each case. Study entry criteria consisted of prematurity and cranial ultrasound documentation of IVH with progressive PHH. As SRCMC is a referral center for several neonatal intensive care units, initial treatment varied according to the referring neonatologist's preference. Acetazolamide and/or serial lumbar punctures were used in some, but not all cases. Each infant was assigned a grade based on the severity of the hemorrhage: grade 1 - hemorrhage confined to the germinal matrix; grade 2 - IVH without ventricular distention (initially); grade 3 - IVH filling and dilating the ventricular system, and grade $4-$ IVH with parenchymal extension $[5,6]$. Variables recorded consisted of gestational age, weight at birth, weight at time of surgery, IVH grade, day of life the hemorrhage occurred, time from hemorrhage to treatment, infection, pretreatment cerebrospinal fluid (CSF) red blood cell (RBC) count, CSF RBC count at termination of urokinase treatment, pretreatment CSF protein level, and CSF protein level at termination of urokinase treatment.

Of the 18 treated infants, 11 were male and 7 female (table 1 ). The average gestational age was 27 weeks (range 23-33). The mean birth weight was $998 \mathrm{~g}$ (range $580-1,830 \mathrm{~g}$ ). The mean weight at time of surgery was $987 \mathrm{~g}$ (range $557-1,830 \mathrm{~g}$ ). Eight infants had a grade 3 and 10 a grade 4 IVH. Most hemorrhages occurred soon after delivery. The mean time at which the hemorrhage was detected was 5 days of life (range 1-15). The mean time from hemorrhage to treatment was 12 days (range $2-35$ ).

\section{Treatment Protocol}

Each infant underwent surgical placement of a ventricular access device (VAD). The treatment period was 7 days during which the VAD was tapped twice daily and urokinase instilled. In the morning, approximately $10-15 \mathrm{~cm}^{3} / \mathrm{kg}$ of CSF was removed. In the afternoon, an amount of CSF was removed equal to the volume of urokinase given. As there were no deleterious effects from the initial low dosage, the total amount of urokinase given was increased during the study to ascertain whether increasing dose would cause increasing beneficial effect. The first 2 infants received 110,000 units, the third 130,000 units, the next 6140,000 units and the final 9280,000 units. Two treatment groups were compared: the 9 infants who received 280,000 units constituting a 'high'-dose group and the other infants a 'low'-dose group. A cranial ultrasound and serum levels of fibrinogen and fibrin degradation products were obtained every other day during the treatment period. CSF was sent daily for determinations of glucose, protein, cell count and culture.

A ratio of the lateral ventricular width and hemispheric width (LVW/HW) at the level of the foramen of Monro was obtained from the coronal images of the pretreatment cranial ultrasound. Normal mean $\mathrm{LVH} / \mathrm{HW}$ has been demonstrated to be 0.32 with $95 \%$ confidence limits of $0.23-0.42$ [7]. This ratio was also obtained posttreatment, either just prior to shunt placement or 1 month following termination of treatment if a shunt was not required.

\section{Shunt Criteria}

After completion of the urokinase treatment, the infants were followed with serial cranial and neurologic examinations and cranial ultrasounds. When the infant reached a weight of $2 \mathrm{~kg}$ a shunt was placed if there were continued signs of increased intracranial pressure (full fontanel, rapid increase in head circumference) and ultrasound evidence of ventriculomegaly.

\section{Historical Control Group}

To establish an expected rate of shunt placement without urokinase treatment, the outcomes of all premature infants who sustained an IVH, developed PHH, and who underwent placement of a VAD at SRCMC and NSH between 1988 and 1992 were retrospectively reviewed. Selection criteria for VAD placement and shunt insertion as well as the amount of CSF removed daily was the same as for the experimental group. To maintain consistency with the experimental 
Table 1. Experimental population, descriptive data and outcome

\begin{tabular}{|c|c|c|c|c|c|c|c|}
\hline \multirow[t]{2}{*}{ Patient } & \multirow[t]{2}{*}{ Grade } & \multirow{2}{*}{$\begin{array}{l}\text { Gestational } \\
\text { age, weeks }\end{array}$} & \multirow{2}{*}{$\begin{array}{l}\text { Birth } \\
\text { weight, } g\end{array}$} & \multirow{2}{*}{$\begin{array}{l}\text { Time to } \\
\text { treatment } \\
\text { days }\end{array}$} & \multicolumn{2}{|c|}{ LVH/HW } & \multirow[t]{2}{*}{ Shunt } \\
\hline & & & & & pre & post & \\
\hline \multicolumn{8}{|c|}{ Low dose } \\
\hline MS & 4 & 26 & 1,130 & 10 & 0.69 & 0.36 & No \\
\hline $\mathrm{KB}$ & 4 & 26 & 910 & 5 & 0.45 & 0.50 & No \\
\hline DK & 3 & 32 & 1,490 & 11 & 0.53 & 0.39 & No \\
\hline $\mathrm{CD}$ & 3 & 26 & 911 & 7 & 0.56 & 0.39 & No \\
\hline $\mathrm{HB}$ & 4 & 28 & 1,130 & 21 & 0.55 & 0.48 & Yes \\
\hline TH & 3 & 33 & 1,440 & 4 & 0.47 & 0.39 & No \\
\hline $\mathrm{RH}$ & 3 & 29 & 1,270 & 12 & 0.57 & 0.51 & Yes \\
\hline HP & 3 & 28 & 870 & 11 & 0.49 & 0.38 & No \\
\hline CR & 4 & 23 & 557 & 10 & 0.58 & 0.49 & Died \\
\hline \multicolumn{8}{|c|}{ High dose } \\
\hline BB & 4 & 24 & 635 & 36 & 0.73 & 0.63 & Yes \\
\hline MK & 3 & 26 & 880 & 17 & 0.61 & 0.62 & Yes \\
\hline TF & 4 & 26 & 735 & 9 & 0.64 & 0.50 & Yes \\
\hline DS & 4 & 27 & 980 & 14 & 0.56 & 0.56 & Yes \\
\hline JP & 3 & 24 & 640 & 8 & 0.56 & 0.51 & Yes \\
\hline $\mathrm{CS}$ & 3 & 32 & 1,730 & 19 & 0.47 & 0.46 & Yes \\
\hline $\mathrm{CS}$ & 4 & 27 & 580 & 20 & 0.50 & 0.45 & Yes \\
\hline $\mathrm{PH}$ & 3 & 26 & 920 & 8 & 0.59 & 0.53 & Yes \\
\hline NW & 4 & 24 & 660 & 15 & 0.68 & 0.59 & Yes \\
\hline
\end{tabular}

group, only infants with grade 3 and 4 hemorrhages were included in the control group: 20 were grade 3 , and 19 were grade 4 . Average gestational age was 28.4 weeks (range $23-33$ ). Average birth weight was 1,086 (range $510-1,800$ ).

\section{Statistical Analysis}

Comparisons between any two groups (i.e., shunt required vs. no shunt required) with categorical data were performed by $\chi^{2}$ analysis. Comparison between groups of continuous or ranked data were analyzed by the t-test statistic with Bonferroni correction, which adjusts $\alpha$ levels for the numerous analyses conducted. Pretreatment to posttreatment comparisons (i.e., change in mean LVW/HW ultrasound measurement) were analyzed by the paired t-test statistic with Bonferroni correction. All analyses used a 0.05 probability level for significance.

\section{Results}

There were no serious deleterious effects related to the urokinase administration. Specifically, recurrent IVH, systemic hemorrhage, or allergic reaction did not occur. One infant developed fever and chills following administration of the urokinase. This reaction has been reported with the intravenous use of urokinase and is not felt to be allergic in origin [A. Sasahara, Abbott Laboratories, pers. commun.]. The infant was given diphenhydramine and acetaminophen prior to treatment and the reaction did not recur. Daily determinations of CSF RBC counts occasionally demonstrated a rise during treatment, but, as cranial ultrasound examination did not demonstrate recurrent hemorrhage, this was posited to be caused by mobilization of RBC's following clot lysis. Serum fibrinogen and fibrin degradation product levels remained within normal levels or did not vary from preoperative baseline levels. One infant (low-dose group) born at 23 weeks' gestation died of respiratory distress syndrome 1 month following completion of urokinase treatment.

Statistical analysis demonstrated no significant differences in the demographic data when the high- and lowdose groups were compared. Four infants developed VAD infections, 22\% (Staphylococcus epidermidis 2, Staphylococcus aureus 1 , Pseudomonas 1 ). There was 1 infection in the low-dose and 3 infections in the high-dose group. All of the infants with VAD infections required shunt placement.

No statistically significant differences were present between pre- and posttreatment $(\mathrm{t}=0.46, \mathrm{p}<0.65 ; \mathrm{t}=$ $-0.51, \mathrm{p}<0.62)$ or between low- and high-dose groups for levels of CSF protein or RBC count at either the initial $(\mathrm{t}=-1.19, \mathrm{p}<0.25 ; \mathrm{t}=-1.13, \mathrm{p}<0.27)$ or follow-up $(\mathrm{t}=-1.52, \mathrm{p}<0.15 ; \mathrm{t}=1.11, \mathrm{p}<0.28)$ evaluation. 
For the group as a whole, pretreatment LVH/HW was 0.57 (range $0.45-0.73$ ) and posttreatment LVH/HW was 0.48 (range $0.36-0.63$ ); this difference is significant $(\mathrm{t}=$ $-4.04, \mathrm{p}<0.001)$. When this ratio is calculated for the low- and high-dose groups, there is a significant difference between pre- and posttreatment values for the low-dose (pre 0.54 , post $0.42(\mathrm{t}=3.10, \mathrm{p}<0.018)$ ) and for the highdose group (pre 0.59 , post $0.54(\mathrm{t}=-3.08, \mathrm{p}<0.011)$ ). Comparison between the low- and high-dose groups showed no difference pretreatment $(\mathrm{p}=0.21)$, but the lowdose group did have a greater reduction posttreatment than the high-dose group $(\mathrm{p}=0.002)$.

Of the 18 survivors, $12(71 \%)$ required shunt placement. For the low-dose group, 3 of $8(37 \%)$ required shunt placement and for the high-dose group all 9 required shunt placement. Within the initial 24 months following shunt placement, 8 shunt revisions were performed $(0.67$ revisions per shunted child).

None of the variables listed above, including presence of infection $\left(\chi^{2}=-2.57, p=0.11\right)$ and duration of time from hemorrhage to treatment $(t=1.19, p>0.25)$, were predictive of treatment success (lack of shunt) with the exception of membership in the low-dose treatment group $\left(\chi^{2}=9.00, \mathrm{p}<0.003\right)$.

\section{Historical Control Group}

Three infants ( $8 \%$ ) had a shunt infection; all 3 subsequently required shunt placement. Three infants died prior to shunt placement. Thirty-three of the 36 survivors required shunt placement $(92 \%)$. Within 2 years of shunt placement, the shunt revision rate was 1.5 revisions/ patient. Comparison of the shunt placement rate betwen the historical control and the urokinase treatment group approaches, but does not reach statistical significance $(\mathrm{p}<$ 0.06 ). When the subgroups were compared with the historical control group, there was a reduction in shunt placement for the low-dose $(\mathrm{p}<0.002)$, but not the high-dose group. For those infants who required shunt placement, the shunt revision rate in the initial 24 months following shunt placement was lower in the treatment group than in the control group $(\mathrm{t}=2.65, \mathrm{p}<0.05)$. The reduction in shunt revision rate occurred in both the low- and highdose groups.

\section{Discussion}

\section{Clinical Course of $\mathrm{PHH}$}

The incidence of $\mathrm{PHH}$ following IVH varies directly with the severity of the hemorrhage: $55 \%$ in grade 3 and $80 \%$ in grade 4 [8]. While in many cases the hydrocephalus will either spontaneously resolve or arrest, the reported incidence varies widely in the literature. In the pediatric and neonatal literature, in which all premature infants who sustain an IVH are included, the rate of shunt placement is $20 \%$ [8]. In the neurosurgical literature the rate is considerably higher, $84-87 \%[9,10]$. The difference is due to referral bias - the neurosurgeon is typically involved only after initial treatment measures such as lumbar puncture or acetazolamide have failed and the ventriculomegaly is progressive. Thus, it is not unexpected that the rate of shunt placement would be high for those infants who have required a neurosurgical consultation.

Accordingly, the outcomes of premature infants treated with urokinase should be compared to a similar group also referred for neurosurgical treatment but not treated with urokinase. The historical control group consists of preterm infants with $\mathrm{PHH}$ following IVH treated with placement of a VAD during a 4-year period prior to beginning this study. The historical comparison group was similar to the treatment group regarding grade of IVH, gestational age, and birth weight. The $92 \%$ incidence of shunt placement in this group is consistent with that reported in the literature from other neurosurgical series concerning the treatment of $\mathrm{PHH}$ of prematurity [10].

\section{Treatment of $\mathrm{PHH}$}

The initial management of $\mathrm{PHH}$ is usually performed by neonatologists and usually consists of observation by serial cranial ultrasounds. If the course of the $\mathrm{PHH}$ is progressive, serial lumbar punctures may be used to control intracranial pressure and to remove bloody CSF. To be effective, the hydrocephalus must be communicating and the taps must be high volume $\left(10-15 \mathrm{~cm}^{3} / \mathrm{kg}\right)$ [8]. In our experience, maintaining a high-volume tap over several days or weeks is not usually technically feasible. Acetazolamide, a carbonic anhydrase inhibitor, may be used but has the side effect of producing a metabolic acidosis. In addition, myelin production may be inhibited as its formation uses a carbonic anhydrase [8].

If the neurosurgeon is involved early in the care of $\mathrm{PHH}$, shunt placement is usually not performed as blood in the CSF will cause catheter obstruction and the imma- 
ture immune system of the premature infant makes infection more likely. This has led to shunt revision rates of 4.2 revisions/patient and infection rates of $50 \%$ when early shunting was attempted as a treatment of $\mathrm{PHH}$ in the premature infant [11]. In an attempt to delay shunt placement, other neurosurgical interventions such as serial ventricular taps and external ventricular drains have been used, but they have limitations of porencephaly and high infection rate, respectively. An alternative method is serial tapping of a subgaleal ventricular reservoir or VAD. Marlin et al. [10] reported a series of premature infants treated with a VAD with significant improvement in both the rates of infection and shunt revision. While the primary purpose of a VAD is to facilitate removal of CSF and blood products, it can also function as a conduit for the instillation of intraventricular medications.

\section{Thrombolytic Therapy}

Dissolution and clearance of the intraventricular blood would have several potential beneficial effects. Most importantly, permanent hydrocephalus may be prevented if rapid clot lysis increases the clearance of fibrin particulate matter that can plug the arachnoid granulations or if decreasing the time the blood is in contact with the meninges lessens the scarring and fibrosis of the basilar cisterns. In addition, because fibrin debris frequently obstructs the shunts in those infants who do require shunt placement, clot lysis and clearance of the fibrin debris may decrease the incidence of shunt revision.

Among the thrombolytic agents currently in use, most experience with intraventricular installation has been with urokinase. Urokinase is an enzyme produced by the kidney and found in the urine. The commercially available product, Abbokinase (Abbott Laboratories, North Chicago, Ill., USA), is obtained from human kidney cells by tissue culture techniques. Urokinase activates the endogenous fibrinolytic system by converting plasminogen to plasmin, and plasmin then lyses the fibrin clot. The primary clinical application of urokinase is the restoration of patency of intravenous catheters occluded by blood or fibrin. The most significant adverse reaction to urokinase is bleeding. Allergic reactions are unusual and rare.

Pang et al. [12-14], in 1986, performed a series of experiments in a canine model on the dosage, safety and efficacy of urokinase in the clearance of intraventricular blood and prevention of progressive $\mathrm{PHH}$. In vitro studies showed the minimal dose of urokinase necessary to lyse $10 \mathrm{~cm}^{3}$ of clotted blood was $10,000 \mathrm{IU}$. They then injected 20,000 IU of urokinase (empirically doubling the dose to compensate for clearance of the drug by CSF) eve-

Intraventricular Hemorrhage-Urokinase ry $12 \mathrm{~h}$ for 4 days into the ventricles of 6 animals through an implanted catheter. They found this dose did not cause intracranial hemorrhage, even though the animals had recently undergone brain surgery to place the ventricular catheter. Pathologic examinations of the brains demonstrated no deleterious effects of the urokinase, specifically no acute or chronic inflammation of the brain, ependymal surface or meninges. Autologous, clotted blood was then injected into the ventricles of the animals to create a model of IVH and PHH. In control dogs (those not treated with urokinase), complete lysis of the clot took 38-65 days and $80 \%$ developed progressive ventricular enlargement. The final ventricular volume was up to 14 times the baseline volume. Pathologic examination demonstrated basal subarachnoid fibrosis and extensive ependymal and subependymal damage in the lateral ventricular walls of the hydrocephalic dogs. Treated animals received $20,000 \mathrm{IU}$ of intraventricular urokinase every $12 \mathrm{~h}$ with the first dose given $6 \mathrm{~h}$ after the blood cast preparation. Treatment continued until clots were no longer detected in the ventricles on CT. Clot lysis occurred in 3-6 days with no intracranial or systemic hemorrhages. The neurologic status of the dogs improved, the ventricles decreased in size, and only 2 animals had persistently large ventricles. Pathologic examination showed minimal damage and fibrosis, significantly less than control animals. While this experimental model of IVH is not completely analogous to the germinal matrix hemorrhage in preterm infants, and the canine fibrinolytic system may differ from that of humans, these studies certainly raised the possibility that urokinase might have a beneficial impact on the prevention of $\mathrm{PHH}$ following IVH in the preterm infant.

Urokinase has been used to treat IVH in the adult population [15, 16]. Efficacy in terms of clot lysis and improved outcomes were reported with no adverse reactions to the treatment. San Frutos et al. [17] reported the successful use of urokinase in a 23-day-old infant who was developing loculations in her ventricles after treatment of meningitis/ventriculitis. They demonstrated disappearance of the intraventricular strands following treatment. This raises the intriguing possibility that urokinase may be able to act directly to prevent or diminish the arachnoiditis that causes hydrocephalus following IVH.

Streptokinase, another thrombolytic agent, has been studied by Whitelaw et al. [18]. They treated 9 premature infants with progressive $\mathrm{PHH}$ following IVH. Treatment began 8-27 days after birth. The treatment regimen consisted of 20,000-25,000 units of streptokinase/24 h for 23 days instilled into the ventricles via an occipital cathe-

Pediatr Neurosurg 1997;26:281-287 285 
ter. A lumbar puncture was performed during the streptokinase infusion to help the streptokinase spread from the ventricles to the subarachnoid space. One infant had 'minor' rebleeding resulting in discontinuation of the treatment but no deleterious effects. Although length of followup is not given, all the infants survived and only 1 has required a shunt. In comparison to a previously published multicenter study [19] of tapping versus conservative management of PHH that reported a shunt rate of $62 \%$, the authors found their results to be encouraging.

The above are noncontrolled clinical series with relatively small numbers of patients and limited follow-up, but, in a fashion similar to the experimental studies of Pang et al. [12-14], suggest that intraventricular thrombolytic therapy is safe and effective in preventing hydrocephalus after IVH.

\section{Intraventricular Urokinase}

Safety. At the doses used in this study, no significant deleterious events occurred. Cranial ultrasounds, performed every other day during treatment, showed no evidence of rebleeding. No allergic reactions occurred. Parameters of clot lysis (fibrin degradation products, fibrinogen levels) were assayed every other day during treatment and no changes from baseline levels occurred.

Efficacy. A statistically significant reduction of the LVW/HW ratio occurred following treatment both for the group as a whole and for the two dosage groups considered individually. There was, however, a significantly greater reduction in the ratio following treatment of the low-dose as compared to the high-dose group. The beneficial effects of low-dose urokinase were further demonstrated by a significant reduction in the rate of shunt placement for this group when compared to the historical control group. This beneficial effect was not demonstrated for the highdose group in which all 9 infants required shunt placement. Empirically, we had anticipated outcome would continue to improve (fewer shunts) as the dose of urokinase was increased. A decremental response was not expected. While increasing the dose of urokinase may saturate the reaction that converts plasminogen to plasmin, excess urokinase should not hinder the reaction [J. Hankin, Abbott Laboratories, pers. commun.]. The difference in outcome between the high- and low-dose groups may simply be a sampling error due to the small numbers in each group.

A potential benefit of intraventricular urokinase administration may be reduction in the shunt revision rate for those infants requiring shunt placement. The shunt revision rate was significantly lower in the urokinase treatment group (both low- and high-dose patients) when compared to the historical control group even though the follow-up period was longer for the treatment group. Due to the intraventricular blood and fibrin as well as the presence of adhesions and scarring within the ventricles, shunt revisions are common in this patient population. Urokinase may speed clot dissolution, prevent the formation of fibrin adhesions and thus reduce the shunt malfunction rate.

\section{Predictive Variables}

Other than membership in the low-dose group, no factors predictive of success or failure of treatment were identified. This also may be a reflection of the small numbers in this series. There are several factors that, even though not found to influence outcome in this study, might eventually prove to have an impact on whether treatment is successful, inlcuding time from hemorrhage to treatment, severity of hemorrhage, and occurrence of infection.

Experience generated by studies using urokinase to treat pulmonary emboli has demonstrated that thrombi older than 5-7 days are resistant to lysis [14]. While intraventricular clot lysis may vary considerably from intravascular clot lysis, it does seem likely that early intervention will be more effective. However, as many infants will resolve the IVH without $\mathrm{PHH}$, treatment with urokinase should be delayed until ventricular enlargement is present. This may prove to be a major obstacle to successful treatment of $\mathrm{PHH}$ with urokinase because, as discussed above, ventricular enlargement commonly does not occur until 1-3 weeks following the hemorrhage. Thus, by the time treatment is instituted, the clot may no longer be susceptible to lysis by the medication.

It seems intuitive that increasing volumes of blood within the ventricles would require increasing amounts of urokinase to effect lysis and clearance. However, lack of knowledge concerning intraventricular urokinase pharmacokinetics makes this uncertain. While Pang et al. [12] have demonstrated that in vitro the amount of urokinase necessary to lyse a specific amount of blood can be determined, in vivo determination of optimal dose is difficult because of clearance of the drug by CSF flow, abnormal CSF flow that may prevent the urokinase from reaching all the surface area of the clot, and the presence of plasminogen activator inhibitors [20]. Our findings of better response at lower doses underlines this uncertainty, suggesting the lack of a predictable linear dose response.

While it did not reach statistical significance as a predictive variable, every infant whose course was compli- 
cated by a VAD infection required shunt placement. The inflammatory response within the ventricles caused by infection is likely to increase the incidence of permanent hydrocephalus. The relatively higher incidence of infection within the high-dose group may have contributed to the higher rate of shunt placement in this group. The infection rate in the treatment group is higher than in the historical control group, possibly related to the twice-daily tap schedule (during the 7-day treatment period) in the treatment group versus once a day for the contrast group. The inevitable occurrence of infection and resultant morbidity should be factored into the risk of this treatment regimen.

\section{Conclusion}

While the initial results with a higher dose urokinase have been disappointing, there appears to be benefit with 10,000 units. In addition, for those infants requiring shunts, both treatment groups demonstrated fewer shunt revisions than the historical contrast group. The use of intraventricular urokinase, in the doses used in this study, has not been associated with rehemorrhage or allergic reactions.

\section{References}

1 Volpe JJ: Neonatal intraventricular hemorrhage. N Engl J Med 1981;303:886-890.

2 Erashin Y, McLone DG, Storrs BB, et al: Review of 3,017 procedures for the management of hydrocephalus in children. Concepts Pediatr Neurosurg 1989:9:21-28.

3 Klein DM: Shunt Infections. Concepts in Neurosurgery. Baltimore, Williams \& Wilkins, 1990 , vol 3.

4 Larroche JC: Post-hemorrhagic hydrocephalus in infancy: Anatomical study. Biol Neonate 1972;20:287-299.

5 Duncan CC, Ment LR, Ogle E: Acquired problems of the newborn; in McLaurin RL, Schut L, Venes JL, Epstein F (eds): Pediatric Neurosurgery. Philadelphia, Saunders, 1989, pp 230237

6 Papile L, Burstein J, Burstein R, Koffler H: Incidence and evolution fo subependymal and intraventricular hemorrhage: A study of infants with birth weights less than $1,500 \mathrm{~g}$. J Pediatr 1978:92:529-534.

7 Poland RL, Slovis TL, Shankaran S: Normal values for ventricular size as determined by real-time sonographic techniques. Pediatr Radiol 1985;15:12-14.
8 Volpe JJ: Intracranial Hemorrhage: Germinal Matrix-Intraventricular Hemorrhage of the Premature Infant. Neurology of the Newborn. Philadelphia, Saunders, 1995.

9 Hudgins RJ, Boydston WR, Hudgins PA, et al: Treatment of intraventricular hemorrhage in the premature infant with urokinase: A preliminary report. Pediatr Neurosurg 1994;20:190197.

10 Marlin AE, Rivera S, Gaskill SJ: Treatment of posthemorrhagic ventriculomegaly in the preterm infant: Use of the subcutaneous ventricular reservoir. Concepts Pediatr Neurosurg 1988:8:15-22.

11 Boynton BR, Boynton CA, Merritt TA, Vaucher YE, et al: Ventriculoperitoneal shunt in low birth weight infants with intracranial hemorrhage: Neurodevelopmental outcome. Neurosurgery 1986;18:141-145.

12 Pang D, Sclabassi RJ, Horton JA: Lysis of intraventricular clot with urokinase in a canine model. 1. Canine intraventricular blood cast model. Neurosurgery 1986;19:540-546.

13 Pang D, Sclabassi RJ, Horton JA: Lysis of intraventricular clot with urokinase in a canine model. 2 . In vivo safety study of intraventricular urokinase. Neurosurgery 1986;19:547-552.

14 Pang D, Sclabassi RJ, Horton JA: Lysis of intraventricular clot with urokinase in a canine model. 3. Effects of intraventricular urokinase on clot lysis and posthemorrhagic hydrocephalus. Neurosurgery 1986;19:553-572.
15 Shen PH, Matsuoka Y, Kawajiri K, et al: Treatment of intraventricular hemorrhage using urokinase. Neurol Med Chir (Tokyo) 1990;30: 329-333.

16 Todo T, Usui M, Takakura K: Treatment of severe intraventricular hemorrhage by intraventricular infusion of urokinase. J Neurosurg 1991;74:81-86.

17 San Frutos MA, Fernandez-Pavon A, PerezHigueras A, et al: Local urokinase for the treatment of ventriculitis complications. Acta Paediatr Scand 1986;75:497-498.

18 Whitelaw A, Rivers RPA, Creighton L, et al: Low-dose intraventricular fibrinolytic treatment to prevent posthemorrhagic hydrocephalus. Arch Dis Child 1992;67:12-14.

19 Ventricular Trial Group: Randomized trial of early tapping vs. conservative management in infants with posthaemorrhagic hydrocephalus. Arch Dis Child 1990;65:3-10.

20 Rao JS, Chen M, Festoff BW: Plasminogen activator inhibitor 1 , the primary regulator of fibrinolysis, in normal human cerebrospinal fluid. J Neurosc Res 1993;34:340-345. 